\title{
BENEFICIOS DE LA ACTIVIDAD FÍSICA EN ESPACIOS NATURALES SOBRE LA SALUD DEL ADULTO MAYOR
}

\section{HEALTH BENEFITS OF GREEN PHYSICAL ACTIVITY FOR OLDER ADULTS}

\author{
Hannia Reyes-Rincón (1), Yolanda Campos-Uscanga (2) \\ (1) Becaria del Sistema Nacional de Investigadores, Instituto de Salud Pública. México. \\ (2) Instituto de Salud Pública, Universidad Veracruzana \\ Investigadora de tiempo completo del Instituto de Salud Pública. México.
}

\footnotetext{
Reyes-Rincón, H., \& Campos-Uscanga, Y. (2020). Beneficios de la actividad física en espacios naturales sobre la salud del adulto mayor. Revista Ene De Enfermería, 14(2). Consultado de http://www.ene-enfermeria.org/ojs/index.php/ ENE/article/view/968
} 


\section{Resumen}

El envejecimiento es un hecho irreversible que actualmente ha cobrado relevancia por el aumento de la esperanza de vida y con ello la proporción de adultos mayores que requieren estrategias para tener adecuada calidad de vida. Existe evidencia de que dos factores favorecen la calidad de vida en distintos grupos poblacionales: la actividad física y el contacto con espacios naturales. Por tal motivo, se realizó una revisión sistemática para identificar las motivaciones de los adultos mayores para realizar actividad física en espacios naturales y los efectos que esta tiene en su salud. Los hallazgos sugieren que aunque existen barreras como falta de tiempo, inseguridad pública y ausencia de espacios idóneos, también hay condiciones que favorecen su realización, tales como mayor conexión con la naturaleza, aumento en la cohesión social y mejora en la seguridad en sí mismos. La actividad física en espacios naturales mejora el bienestar psicosocial, aumenta la adherencia al ejercicio, incrementa la función física y psicológica y disminuye las tasas de mortalidad en este grupo de edad. Por lo tanto, el "ejercicio verde" puede ser una buena estrategia para favorecer la calidad de vida en el adulto mayor.
Palabras clave: adulto mayor, actividad física, terapia por ejercicio, naturaleza, promoción de la salud

\section{Abstract}

Aging is an irreversible fact that nowadays has become relevant for the increase of life expectancy and with it the old adult who require strategies to have right life quality. There are evidences that two factors favor life quality in different population groups: physical activity and the contact with natural environment. For that reason, it was made a systematical review to identify motivation of old adults to do physical activity in natural environment (spaces) and the effects of in their health. The findings suggest that although there are barriers like insecurity and lack of appropriate spaces, there are also favorable conditions to do it, such as major connection with nature, increase in social cohesion and more self confidence. Physical activity in natural environments improves psychosocial well-being, increases exercise adherence, favors a major moving capacity and decreases mortality rate in this age group. Therefore, green exercise can be a good strategy to favor life quality in old adults.

Key words: older adult, physical activity, exercise therapy, nature, health promotion 


\section{INTRODUCCIÓN}

La mayoría de los países del mundo están experimentando un aumento en la proporción de personas mayores (1), por lo que el envejecimiento está por convertirse en una de las transformaciones sociales más significativas del siglo $X X I$ (2). Según el informe "Perspectivas de la Población Mundial", se espera que el número de personas mayores, se duplique para 2050 y triplique para 2100 (3).

El envejecimiento de la población puede considerarse un éxito de las políticas de salud pública y el desarrollo socioeconómico. Sin embargo, también constituye un reto para mejorar al máximo la salud y la capacidad funcional, así como la participación social y seguridad de las personas mayores (4).

Dentro de las estrategias para mantener la calidad de vida del adulto mayor resalta la actividad física que ha probado generar cambios positivos en equilibrio, fuerza muscular, capacidad de ambulación del adulto mayor (5), rendimiento físico, aumento de la memoria y atención, mayor equilibrio, coordinación e interacción social (6), mayor masa ósea y muscular, disminución del riesgo de padecer osteoporosis y mejor pronóstico de recuperación (7), así como aumento de los niveles plasmáticos de vitamina $\mathrm{D}$ (8).

Por otra parte, se ha documentado que tener contacto con espacios natu- rales genera beneficios para la salud física y mental (9). Dentro de los espacios naturales que se han estudiado en términos de sus contribuciones a la salud, los que más destacan son los espacios verdes, entendidos como terreno parcial o totalmente cubiertos por pasto, árboles, arbustos o cualquier otro tipo de vegetación, como parques, jardines comunitarios, patios de escuela, etc. (10). También se han estudiado los espacios azules, es decir, entornos al aire libre, naturales o artificiales, que cuentan con gran cantidad de agua y son accesibles a los seres humanos de manera proximal (estando en, sobre o cerca del agua) o distalmente (pudiendo ver, oír o sentir el agua de otra manera) (11).

Contar con espacio verde en el área de residencia de un individuo está relacionado con el aumento de la felicidad y la satisfacción con la vida (12), a su vez ofrecen más posibilidades de realizar actividad física y, por consiguiente, volverse personas activas (13). En madres solteras que suelen pasear, hacer ejercicio, observar aves y conversar en espacios verdes, dichas actividades son mediadoras entre el estrés laboral y la relación con la familia (14). También se ha observado que los espacios naturales contribuyen a superar dificultades emocionales y de comportamiento en niños y síntomas depresivos en adolescentes y 
adultos jóvenes (15), lo mismo que ocurre con los espacios azules que mejoran el bienestar psicosocial, convirtiéndose en áreas clave para la promoción de la salud pública (16).

De acuerdo con lo antes revisado, realizar actividad física al aire libre en adultos disminuye los niveles de ansiedad y favorece el bienestar psicológico (17) y cuando se realiza en espacios naturales genera una mayor atención dirigida y aumenta las interacciones sociales (18). Por lo que en adultos la actividad física que se realiza en espacios al aire libre tiene un mayor efecto sobre el bienestar mental que cuando se realiza en espacios cerrados (19).

Lo anterior permite suponer que la actividad física y los espacios naturales en conjunto pueden brindar beneficios potenciados a la salud de los adul- tos mayores. Por tal motivo el propósito del presente estudio fue realizar una búsqueda sistemática para identificar las motivaciones de este sector de la población para realizar actividad física en espacios naturales y los efectos que esta tiene en su salud.

\section{MATERIAL Y MÉTOdO}

Se realizó una búsqueda exhaustiva en 2 bases de datos: Pubmed y Scielo, de diciembre del 2018 a febrero del 2019. Los términos de búsqueda fueron "adulto mayor", "espacios verdes", "espacios azules", y "actividad física". La estrategia de búsqueda se define en la Tabla 1.

Tabla 1. Estrategia de búsqueda de información

\begin{tabular}{|l|l|}
\hline Base de datos & $\begin{array}{l}\text { Búsqueda académica en Pubmed y Scielo con textos } \\
\text { completos. }\end{array}$ \\
\hline Palabras clave & $\begin{array}{l}\text { Se emplearon los siguientes términos de los descriptores en } \\
\text { salud en español y su traducción al inglés: adulto mayor, } \\
\text { actividad física y espacios verdes. } \\
\text { Además se utilizaron las siguientes combinaciones: espacios } \\
\text { verdes y salud, beneficios de los espacios verdes, adulto } \\
\text { mayor y espacios verdes, ejercicio verde, actividad física en el } \\
\text { exterior, ejercicio verde en la vejez, actividad física en el } \\
\text { exterior, ejercicio al aire libre, actividad física al aire libre y } \\
\text { adulto mayor. }\end{array}$ \\
\hline
\end{tabular}


Criterios de inclusión

1. El estudio investigó una o dos variables de interés.

2. El estudio podía ser transversal, longitudinal o experimental.

3. La población estudiada está compuesta por adultos mayores de 40 años de edad, sin importar sexo, nivel socioeconómico, lugares de procedencia, nivel educativo.

4. El estudio fue publicado en una revista científica perteneciente a la base de datos de pubmed y Scielo.

5. El estudio fue publicado en los últimos 5 años.

Extracción de datos, validación y evaluación de calidad

Se elaboró una plantilla de datos para registrar autor, objetivo, tamaño de la muestra, tipo de estudio, medición de la actividad física en los espacios verdes y beneficios tanto físicos como mentales en los adultos mayores, los cuales están plasmados en la sección de resultados.

Cada artículo encontrado tras la búsqueda exhaustiva fue evaluado usando otra plantilla diseñada para identificar calidad, afinidad y coherencia con el tema de interés, haciendo revisión por pares y manteniendo aquellos en que ambas personas consideraban cumplía con los criterios. Los artículos se incluyeron o excluyeron en función de su título, resúmenes y versión completa para obtener la congruencia entre su objetivo, pregunta de investigación y resultados.
Se identificaron 189 artículos en la búsqueda inicial, 169 fueron rechazados por no cumplir alguno de los criterios de inclusión. Solo 20 documentos cumplieron lo establecido.

\section{Resultados}

El adulto mayor prefiere el ejercicio verde porque lo identifica como favorecedor de cohesión social, conexión con la naturaleza, y bienestar físico y mental. Sin embargo, reportan diferentes barreras para su realización como falta de tiempo, lejanía, inseguridad y falta de accesibilidad (Tabla 2).

$$
\text { El ejercicio verde en el adulto }
$$
mayor genera beneficios en bienestar psicológico, autoestima, disfrute con la actividad física, adherencia al ejercicio así como reducción en las tasas de mortalidad y morbilidad, más que cualquier otro ejercicio en interiores (Tabla 3 ). 
Tabla 2. Características, facilitadores y limitantes para la realización de ejercicio verde en los adultos y adultos mayores

\begin{tabular}{|c|c|c|c|c|}
\hline $\begin{array}{l}\text { Autores y } \\
\text { año }\end{array}$ & $\begin{array}{l}\text { Objetivo de } \\
\text { estudio }\end{array}$ & Participantes & Metodología & Resultados \\
\hline $\begin{array}{l}\text { Selby, } \\
\text { Hayes, } \\
\text { O'Sulliva, } \\
\text { O'Neil, } \\
\text { Harmon; } \\
2018(20)\end{array}$ & $\begin{array}{l}\text { Conocer las } \\
\text { percepciones sobre } \\
\text { el ejercicio verde y } \\
\text { las barreras para su } \\
\text { realización como } \\
\text { parte del } \\
\text { tratamiento en } \\
\text { pacientes con dolor } \\
\text { crónico. }\end{array}$ & $\begin{array}{l}113 \text { pacientes } \\
\text { adultos de } 50 \text { a } \\
70 \text { años, que } \\
\text { asistieron a una } \\
\text { clínica de dolor } \\
\text { crónico en } \\
\text { Irlanda. }\end{array}$ & $\begin{array}{l}\text { Estudio } \\
\text { transversal a } \\
\text { través de un } \\
\text { cuestionario } \\
\text { para evaluar } \\
\text { percepción, } \\
\text { accesibilidad y } \\
\text { barreras del } \\
\text { ejercicio verde. }\end{array}$ & $\begin{array}{l}\text { Aunque dijeron estar } \\
\text { dispuestos a realizar } \\
\text { ejercicio verde como una } \\
\text { parte del tratamiento, } \\
\text { identificaron algunas } \\
\text { barreras: la lejanía de } \\
\text { lugares verdes, la poca } \\
\text { disponibilidad de tiempo y la } \\
\text { poca valoración personal de } \\
\text { los beneficios del ejercicio. }\end{array}$ \\
\hline $\begin{array}{l}\text { Calogiuri,El } \\
\text { liott; } 2017 \\
(21)\end{array}$ & $\begin{array}{l}\text { Explorar } \\
\text { comportamientos y } \\
\text { motivaciones para } \\
\text { realizar actividad } \\
\text { física comparando } \\
\text { el ejercicio verde y } \\
\text { el ejercicio en } \\
\text { interiores. }\end{array}$ & $\begin{array}{l}2168 \text { adultos } \\
\text { elegidos } \\
\text { aleatoriamente, y } \\
\text { estratificados por } \\
\text { sexo, edad y área } \\
\text { geográfica, en } \\
\text { Noruega. }\end{array}$ & $\begin{array}{l}\text { Estudio } \\
\text { transversal. Los } \\
\text { datos se } \\
\text { recabaron a } \\
\text { través de una } \\
\text { encuesta } \\
\text { nacional en } \\
\text { línea creada por } \\
\text { Norsk Friluftsliv. }\end{array}$ & $\begin{array}{l}\text { La edad avanzada se asoció } \\
\text { con mayor probabilidad de } \\
\text { realizar ejercicio verde. } \\
\text { Entre los adultos mayores } \\
\text { los motivos principales } \\
\text { fueron la experiencia en la } \\
\text { naturaleza y la convivencia. }\end{array}$ \\
\hline $\begin{array}{l}\text { Calogiuri, } \\
\text { Patil, } \\
\text { Aamodt; } \\
2016 \text { (22) }\end{array}$ & $\begin{array}{l}\text { Examinar en qué } \\
\text { medida el ejercicio } \\
\text { verde es una fuente } \\
\text { común de actividad } \\
\text { física en diferentes } \\
\text { grupos sociales y } \\
\text { evaluar su } \\
\text { importancia para la } \\
\text { equidad en salud. }\end{array}$ & $\begin{array}{l}2168 \text { participantes } \\
\text { elegidos } \\
\text { aleatoriamente y } \\
\text { estratificados por } \\
\text { sexo, edad y área } \\
\text { geográfica, en } \\
\text { Noruega. }\end{array}$ & $\begin{array}{l}\text { Estudio } \\
\text { transversal. Los } \\
\text { datos se } \\
\text { recabaron de la } \\
\text { Encuesta } \\
\text { nacional en } \\
\text { línea creada por } \\
\text { Norsk Friluftsliv. }\end{array}$ & $\begin{array}{l}\text { El ejercicio verde es una } \\
\text { buena estrategia para la } \\
\text { realización de actividad } \\
\text { física en adultos mayores y } \\
\text { la prefieren sobre otro tipo } \\
\text { de ejercicio. Los que viven } \\
\text { en el campo son más } \\
\text { propensos a su realización } \\
\text { en comparación con } \\
\text { aquellos que viven en } \\
\text { grandes ciudades. }\end{array}$ \\
\hline $\begin{array}{l}\text { Pleson, } \\
\text { Nieuwendy } \\
\text { k, Lee, } \\
\text { Chaddah, } \\
\text { Nykiforuk, } \\
\text { Schopfloch } \\
\text { er; } 2014 \\
(23)\end{array}$ & $\begin{array}{l}\text { Comprender el uso } \\
\text { y las percepciones } \\
\text { de los adultos } \\
\text { mayores sobre los } \\
\text { espacios verdes } \\
\text { comunitarios. }\end{array}$ & $\begin{array}{l}\text { Se analizaron } 7 \\
\text { espacios verdes } \\
\text { en Taipei, Taiwán, } \\
\text { contabilizando el } \\
\text { número de } \\
\text { usuarios, de ellos } \\
\text { seleccionaron } 19 \\
\text { participantes } \\
\text { mayores de } 60 \\
\text { años, para la } \\
\text { realización de } \\
\text { entrevistas. }\end{array}$ & $\begin{array}{l}\text { Estudio } \\
\text { cuantitativo y } \\
\text { cualitativo. La } \\
\text { observación de } \\
\text { los espacios } \\
\text { verdes } \\
\text { comunitarios se } \\
\text { hizo a través del } \\
\text { formulario de } \\
\text { codificación del } \\
\text { Sistema para el } \\
\text { Juego de } \\
\text { Observación y } \\
\text { Recreación en } \\
\text { Comunidades. }\end{array}$ & $\begin{array}{l}\text { Los adultos mayores son } \\
\text { quienes más utilizan los } \\
\text { espacios verdes por la } \\
\text { mañana, especialmente } \\
\text { para realizar actividad física } \\
\text { o actividades de recreación, } \\
\text { compromiso social y } \\
\text { optimizar la salud física, } \\
\text { mental y el bienestar. } \\
\text { Estos espacios brindan a los } \\
\text { adultos mayores un sentido } \\
\text { de seguridad en sí mismos, } \\
\text { compromiso social y } \\
\text { actividades de promoción de } \\
\text { la salud. }\end{array}$ \\
\hline
\end{tabular}




\begin{tabular}{|c|c|c|c|c|}
\hline $\begin{array}{l}\text { Finlay, } \\
\text { Franke, } \\
\text { McKay, } \\
\text { Sims- } \\
\text { Gould } \\
2015(24)\end{array}$ & $\begin{array}{l}\text { Evaluar si los } \\
\text { espacios verdes y } \\
\text { azules promueven } \\
\text { la actividad física, } \\
\text { el bienestar mental } \\
\text { y la conexión social } \\
\text { de los adultos } \\
\text { mayores e } \\
\text { identificar las } \\
\text { posibles barreras } \\
\text { para su uso. }\end{array}$ & $\begin{array}{l}27 \text { participantes } \\
\text { de } 65 \text { a } 86 \text { años } \\
\text { pertenecientes al } \\
\text { área } \\
\text { metropolitana de } \\
\text { Vancouver, } \\
\text { Canadá. }\end{array}$ & $\begin{array}{l}\text { Estudio } \\
\text { cualitativo, a } \\
\text { través de una } \\
\text { entrevista, la } \\
\text { primera parte } \\
\text { se llevó a cabo } \\
\text { en un espacio } \\
\text { cerrado y la } \\
\text { segunda } \\
\text { caminando por } \\
\text { el vecindario }\end{array}$ & $\begin{array}{l}\text { Los espacios verdes y } \\
\text { azules tienen un impacto } \\
\text { significativo en la salud } \\
\text { física, mental y social en los } \\
\text { adultos mayores. Sin } \\
\text { embargo la inseguridad o } \\
\text { delincuencia, y la falta de } \\
\text { accesibilidad son barreras } \\
\text { que impiden visitas } \\
\text { frecuentes a estos espacios. }\end{array}$ \\
\hline $\begin{array}{l}\text { Portegijs, } \\
\text { Keskinen, } \\
\text { Tsai, } \\
\text { Rantanen, } \\
\text { Rantakokk } \\
\text { O; } 2017 \\
(25)\end{array}$ & $\begin{array}{l}\text { Evaluar la } \\
\text { accesibilidad a } \\
\text { espacios verdes } \\
\text { para caminar e } \\
\text { identificar } \\
\text { condiciones } \\
\text { ambientales } \\
\text { percibidas como } \\
\text { factores predictivos } \\
\text { de actividad física. }\end{array}$ & $\begin{array}{l}174 \text { adultos de } \\
75-90 \text { años de } \\
\text { edad que vivían } \\
\text { independienteme } \\
\text { nte en el centro } \\
\text { de Finlandia. }\end{array}$ & $\begin{array}{l}\text { E s t u d i o } \\
\text { transversal, } \\
\text { basado en el } \\
\text { p roy e c o } \\
\text { "Características } \\
\text { geográficas, } \\
\text { movilidad al aire } \\
\text { libre y actividad } \\
\text { física de las } \\
\text { pers o n a s } \\
\text { mayores". }\end{array}$ & $\begin{array}{l}\text { Las condiciones } \\
\text { ambientales favorables } \\
\text { percibidas así como la } \\
\text { accesibilidad de los } \\
\text { espacios verdes se } \\
\text { asociaron con mayor } \\
\text { frecuencia de caminar en } \\
\text { adultos mayores con y sin } \\
\text { limitaciones física. }\end{array}$ \\
\hline $\begin{array}{l}\text { Astell-Burt, } \\
\text { Feng, Kolt; } \\
2014 \text { (26) }\end{array}$ & $\begin{array}{l}\text { Examinar si vivir en } \\
\text { vecindarios verdes } \\
\text { se asocia con la } \\
\text { participación en } \\
\text { caminatas y } \\
\text { actividad física } \\
\text { moderada a } \\
\text { vigorosa (AFMV). }\end{array}$ & $\begin{array}{l}203883 \text { adultos } \\
\text { australianos de } 45 \\
\text { años y más. }\end{array}$ & $\begin{array}{l}\text { Estudio } \\
\text { transversal, } \\
\text { basado en la } \\
\text { encuesta } \\
\text { Australia activa. }\end{array}$ & $\begin{array}{l}\text { Los vecindarios más verdes } \\
\text { se asociaron con caminar, } \\
\text { AFMV y mayor frecuencia } \\
\text { de participación en estas } \\
\text { actividades por parte de } \\
\text { adultos mayores. }\end{array}$ \\
\hline $\begin{array}{l}\text { Harada, } \\
\text { Lee, } \\
\text { Sungchul } \\
\text { Lee, Bae, } \\
\text { Harada, } \\
\text { Suzuki, } \\
\text { Shimada } \\
2017 ;(27)\end{array}$ & $\begin{array}{l}\text { Determinar si existe } \\
\text { asociación entre el } \\
\text { tiempo al aire libre } \\
\text { y el funcionamiento } \\
\text { físico y psicológico } \\
\text { considerando el } \\
\text { papel mediador de } \\
\text { la actividad física. }\end{array}$ & $\begin{array}{l}192 \text { individuos } \\
\geq 70 \text { años con } \\
\text { deterioro cognitivo } \\
\text { global } \\
\text { pertenecientes a } \\
\text { Midori-Ward, } \\
\text { Japón }\end{array}$ & $\begin{array}{l}\text { Estudio } \\
\text { transversal. El } \\
\text { tiempo al aire } \\
\text { libre se midió } \\
\text { mediante GPS, } \\
\text { la función física, } \\
\text { la aptitud } \\
\text { cardiorrespirator } \\
\text { ia y la fuerza de } \\
\text { extremidades a } \\
\text { través de las } \\
\text { pruebas de } \\
\text { caminata de } 6 \\
\text { minutos y de } \\
\text { soporte de silla } \\
\text { de cinco } \\
\text { repeticiones. }\end{array}$ & $\begin{array}{l}\text { El tiempo al aire libre se } \\
\text { asoció de forma positiva con } \\
\text { los pasos por día y de forma } \\
\text { inversa con la depresión. A } \\
\text { su vez, los pasos por día } \\
\text { correlacionaron } \\
\text { positivamente con la aptitud } \\
\text { cardiorrespiratoria, la } \\
\text { resistencia de las } \\
\text { extremidades inferiores y el } \\
\text { bienestar. }\end{array}$ \\
\hline
\end{tabular}


REYES-RINCÓN, CAMPOS-USCANGA BENEFICIOS DE LA ACTIVIDAD FÍSICA EN ESPACIOS NATURALES SOBRE LA SALUD DEL ADULTO MAYOR

\begin{tabular}{|c|c|c|c|c|}
\hline $\begin{array}{l}\text { Wu, Jones, } \\
\text { Van Sluijis, } \\
\text { Griffin, } \\
\text { Wareham; } \\
2016(28)\end{array}$ & $\begin{array}{l}\text { Explorar la } \\
\text { importancia de las } \\
\text { características } \\
\text { percibidas y } \\
\text { objetivas } \\
\text { (transitables, uso } \\
\text { correcto del suelo y } \\
\text { conexión con la } \\
\text { calle) del espacio } \\
\text { verde de los } \\
\text { vecindario. }\end{array}$ & $\begin{array}{l}8281 \text { participantes } \\
\text { de } 40 \text { a } 79 \text { años } \\
\text { de edad; } \\
\text { seleccionados a } \\
\text { partir de la } \\
\text { investigación } \\
\text { prospectiva } \\
\text { europea sobre el } \\
\text { cáncer y la } \\
\text { nutrición en } \\
\text { Norfolk Reino } \\
\text { Unido. }\end{array}$ & $\begin{array}{l}\text { Estudio } \\
\text { transversal. La } \\
\text { actividad física } \\
\text { fue } \\
\text { autoinformada y } \\
\text { se empleó un } \\
\text { cuestionario } \\
\text { para medir } \\
\text { aspectos } \\
\text { objetivos y } \\
\text { subjetivos del } \\
\text { entorno vecinal. }\end{array}$ & $\begin{array}{l}\text { Las características } \\
\text { percibidas de los entornos } \\
\text { vecinales tienen influencia } \\
\text { positiva en la actividad } \\
\text { física, pero las mejoras de } \\
\text { infraestructura son más } \\
\text { importantes para apoyar la } \\
\text { actividad física. }\end{array}$ \\
\hline $\begin{array}{l}\text { Cassarino, } \\
\text { Bantry- } \\
\text { White, } \\
\text { Setti; } 2019 \\
(29)\end{array}$ & $\begin{array}{l}\text { Explorar la relación } \\
\text { entre las } \\
\text { características } \\
\text { sensoriales o } \\
\text { cognitivas de los } \\
\text { individuos y las } \\
\text { preferencias de los } \\
\text { espacios al aire } \\
\text { libre para caminar. }\end{array}$ & $\begin{array}{l}112 \text { participantes } \\
\text { sanos } \geq 60 \text { años } \\
\text { de edad, en la } \\
\text { región sur de } \\
\text { Irlanda. }\end{array}$ & $\begin{array}{l}\text { Estudio } \\
\text { transversal, } \\
\text { basado en la } \\
\text { encuesta } \\
\text { Entorno al aire } \\
\text { libre vivido } \\
\text { como } \\
\text { entrenamiento } \\
\text { cerebral } \\
\text { "OutLET" }\end{array}$ & $\begin{array}{l}\text { La forma en que los } \\
\text { individuos perciben las } \\
\text { interacciones sociales en los } \\
\text { lugares donde caminan } \\
\text { influye en las preferencias } \\
\text { de los espacios al aire libre } \\
\text { para caminar.. }\end{array}$ \\
\hline
\end{tabular}

Tabla 3. Beneficios físicos y mentales del ejercicio verde en adultos mayores.

\begin{tabular}{|c|c|c|c|c|}
\hline Autores & $\begin{array}{l}\text { Objetivo de } \\
\text { estudio }\end{array}$ & Participantes & Metodología & Resultados \\
\hline $\begin{array}{l}\text { Silva } \\
\text { Piñeiro, } \\
\text { Mayán } \\
\text { Santos; } \\
2016 \text { (30) }\end{array}$ & $\begin{array}{l}\text { Comparar el } \\
\text { efecto en el } \\
\text { estado de ánimo, } \\
\text { la autoestima y el } \\
\text { disfrute con la } \\
\text { actividad física en } \\
\text { dos programas de } \\
\text { ejercicio físico } \\
\text { para personas } \\
\text { mayores de los } \\
\text { municipios } \\
\text { gallegos de } \\
\text { Arousa-Norte, } \\
\text { Galicia. }\end{array}$ & $\begin{array}{l}\text { Muestra } \\
\text { probabilística de } \\
72 \text { mujeres entre } \\
55-70 \text { años: } \\
\text { Grupo 1: } 36 \\
\text { mujeres. } \\
\text { Grupo 2: } 36 \\
\text { mujeres. }\end{array}$ & $\begin{array}{l}\text { Dos programas de } \\
\text { actividad física con } \\
\text { duración de } 75 \\
\text { sesiones, } 3 \text { semanales. } \\
\text { Grupo 1: programa } \\
\text { proactivo con desarrollo } \\
\text { temático variado, } \\
\text { combinación de } \\
\text { contenidos y en } \\
\text { espacios exteriores } \\
\text { Grupo 2: programa de } \\
\text { actividad física en } \\
\text { espacios cubiertos, la } \\
\text { participante debía } \\
\text { atender la correcta } \\
\text { ejecución y número de } \\
\text { repeticiones. }\end{array}$ & $\begin{array}{l}\text { En ambos programas } \\
\text { de actividad física los } \\
\text { resultados fueron } \\
\text { positivos, sin embargo } \\
\text { en el programa } \\
\text { proactivo se } \\
\text { encontraron mejores } \\
\text { resultados en bienestar } \\
\text { psicológico, autoestima } \\
\text { y disfrute con la } \\
\text { actividad física. }\end{array}$ \\
\hline
\end{tabular}




\begin{tabular}{|c|c|c|c|c|}
\hline $\begin{array}{l}\text { Lacharite, } \\
\text { Lemieux, } \\
\text { Brunelle, } \\
\text { Dionne; } \\
2015 \text { (31) }\end{array}$ & $\begin{array}{l}\text { Comparar los } \\
\text { efectos a largo } \\
\text { plazo de un } \\
\text { programa de } \\
\text { actividad física al } \\
\text { aire libre y otro en } \\
\text { interiores sobre } \\
\text { las respuestas } \\
\text { afectivas y la } \\
\text { adherencia al } \\
\text { ejercicio. }\end{array}$ & $\begin{array}{l}23 \text { mujeres } \\
\text { posmenopáusica } \\
\text { s sanas de } 52 \text { a } \\
69 \text { años de edad } \\
\text { de la provincia } \\
\text { de Quebec, } \\
\text { Canadá } \\
\text { seleccionadas al } \\
\text { azar en: } \\
\text { Grupo 1: } 12 \\
\text { mujeres } \\
\text { Grupo 2: } 11 \\
\text { mujeres. }\end{array}$ & $\begin{array}{l}\text { Dos programas de } \\
\text { entrenamiento físico, } \\
\text { con } 3 \text { sesiones } \\
\text { semanales durante } 12 \\
\text { semanas, de } \\
\text { entrenamiento aeróbico } \\
\text { (30 min) resistencia (15 } \\
\text { min) y enfriamiento. } \\
\text { Grupo 1: parque } \\
\text { agradable y natural al } \\
\text { lado de un cuerpo de } \\
\text { agua, con grandes } \\
\text { árboles y una rica } \\
\text { biodiversidad } \\
\text { Grupo } 2: \text { sala de } \\
\text { reuniones en el Centro } \\
\text { de Investigación sobre el } \\
\text { Envejecimiento. }\end{array}$ & $\begin{array}{l}\text { En comparación con el } \\
\text { ejercicio en interiores, } \\
\text { el ejercicio al aire libre } \\
\text { condujo a mayor } \\
\text { adherencia al ejercicio, } \\
\text { fomentó mayor nivel de } \\
\text { actividad física, redujo } \\
\text { los síntomas de } \\
\text { depresión, mejoró la } \\
\text { valencia afectiva, la } \\
\text { tranquilidad y mantuvo } \\
\text { un mayor compromiso } \\
\text { positivo. }\end{array}$ \\
\hline $\begin{array}{l}\text { King, } \\
\text { Salvo, } \\
\text { Banda, } \\
\text { Ahn, } \\
\text { Chapma, } \\
\text { Gill, } \\
\text { Demons, } \\
\text { Tudor- } \\
\text { Locke, } \\
\text { Rosso, } \\
\text { Pahor, } \\
\text { Frank; } \\
2017 \text { (32) }\end{array}$ & $\begin{array}{l}\text { Explorar los } \\
\text { posibles efectos } \\
\text { moderadores de } \\
\text { la disponibilidad y } \\
\text { accesibilidad de } \\
\text { verdor en los } \\
\text { vecindarios sobre } \\
\text { la actividad física } \\
\text { al aire libre. }\end{array}$ & $\begin{array}{l}400 \text { adultos } \\
\text { mayores } \\
\text { sedentarios de } \\
70 \text { a } 89 \text { años de } \\
\text { edad con riesgo } \\
\text { de discapacidad } \\
\text { de movilidad. } \\
\text { Grupo 1: } 142 \\
\text { mujeres y } 63 \\
\text { hombres } \\
\text { Grupo } 2: 136 \\
\text { hombres y } 59 \\
\text { mujeres }\end{array}$ & $\begin{array}{l}\text { Ensayo aleatorizado, } \\
\text { controlado, con } \\
\text { intervención durante } 12 \\
\text { meses, considerando } \\
\text { como covariable el tipo } \\
\text { de vecindario } \\
\text { Grupo 1: intervención de } \\
\text { actividad física que se } \\
\text { centró en actividades } \\
\text { aeróbicas } \\
\text { Grupo 2: intervención } \\
\text { educativa sobre } \\
\text { envejecimiento } \\
\text { saludable. }\end{array}$ & $\begin{array}{l}\text { Aunque ambos grupos } \\
\text { mejoraron la actividad } \\
\text { física. Los adultos } \\
\text { mayores del grupo de } \\
\text { actividad física se } \\
\text { beneficiaron en mayor } \\
\text { medida incorporando } \\
\text { gradualmente } \\
\text { actividades rutinarias } \\
\text { que implican mayor } \\
\text { movimiento como } \\
\text { jardinería, transporte } \\
\text { activo y caminata y las } \\
\text { realizan durante más } \\
\text { minutos. } \\
\text { Los participantes que } \\
\text { vivían en vecindarios } \\
\text { más compactos } \\
\text { aumentaron el ejercicio } \\
\text { y la caminata de ocio } \\
\text { en menor medida que } \\
\text { los de vecindarios } \\
\text { menos compactos. }\end{array}$ \\
\hline $\begin{array}{l}\text { Cherrie, } \\
\text { Shortta, } \\
\text { Mitchellb, } \\
\text { Taylorc, } \\
\text { Redmondc } \\
\text { ' Thompson } \\
\text { d, Starre, } \\
\text { Dearyf, } \\
\text { Pearcea; } \\
2018 \text { (33) }\end{array}$ & $\begin{array}{l}\text { Examinar si la } \\
\text { disponibilidad de } \\
\text { espacios verdes } \\
\text { se asocia con } \\
\text { cambios } \\
\text { relacionados con } \\
\text { la función } \\
\text { cognitiva. }\end{array}$ & $\begin{array}{l}1091 \\
\text { participantes, } \\
\text { nacidos en } \\
1936, \text { de } \\
\text { Edimburgo y de } \\
\text { los Lothians en } \\
\text { Escocia } 543 \\
\text { mujeres y } 548 \\
\text { hombres. }\end{array}$ & $\begin{array}{l}\text { Estudio longitudinal } \\
\text { retrospectivo; prueba de } \\
\text { Moray House (MHT) la } \\
\text { cual mide la función } \\
\text { cognitiva. Buscando } \\
\text { valorar cambios en la } \\
\text { función cognitiva de los } \\
11 \text { a los } 70 \text { años de } \\
\text { edad y de los } 70 \text { a los } \\
76 \text { años. }\end{array}$ & $\begin{array}{l}\text { Mayor disponibilidad de } \\
\text { espacios verdes tanto } \\
\text { en la infancia como en } \\
\text { la edad adulta se } \\
\text { asoció con el } \\
\text { envejecimiento } \\
\text { cognitivo exitoso. La } \\
\text { creación de parques } \\
\text { públicos en los barrios, } \\
\text { puede ayudar a } \\
\text { ralentizar la tasa de } \\
\text { declive cognitivo en la } \\
\text { edad adulta. }\end{array}$ \\
\hline
\end{tabular}




\begin{tabular}{|c|c|c|c|c|}
\hline $\begin{array}{l}\text { Neale, } \\
\text { Aspinall, } \\
\text { Roe, Tilley, } \\
\text { Mavros, } \\
\text { Cinderby, } \\
\text { Coyne, } \\
\text { Thin, } \\
\text { Bennett, } \\
\text { Thompson } \\
\text {; } 2017 \text { (34) }\end{array}$ & $\begin{array}{l}\text { Comprender el } \\
\text { impacto del } \\
\text { entorno en la } \\
\text { actividad neuronal } \\
\text { mediante el uso } \\
\text { de } \\
\text { electroencefalogr } \\
\text { afía móvil. }\end{array}$ & $\begin{array}{l}95 \text { adultos sanos } \\
\text { de } 65 \text { años o } \\
\text { más, de Leith, } \\
\text { un barrio urbano } \\
\text { histórico en } \\
\text { Edimburgo, } \\
\text { Escocia }\end{array}$ & $\begin{array}{l}\text { Estudio experimental, } \\
\text { caminata de } 10 \text { a } 15 \text { min } \\
\text { Grupo 1: espacios } \\
\text { urbanos verdes } \\
\text { (predominio de } \\
\text { vegetación). } \\
\text { Grupo 2: espacios } \\
\text { urbanos ocupados } \\
\text { (predominio de edificios, } \\
\text { áreas pavimentadas y } \\
\text { tráfico vehicular) } \\
\text { Grupo 3: espacios } \\
\text { urbanos tranquilos } \\
\text { (predominio de edificios, } \\
\text { algunos jardines } \\
\text { delanteros y áreas } \\
\text { pavimentadas, baja } \\
\text { afluencia de tráfico } \\
\text { vehicular). }\end{array}$ & $\begin{array}{l}\text { Se observaron efectos } \\
\text { beneficiosos de } \\
\text { caminar en un entorno } \\
\text { verde urbano como } \\
\text { niveles más altos de } \\
\text { "compromiso" en } \\
\text { comparación con una } \\
\text { calle comercial urbana } \\
\text { y un área residencial } \\
\text { tranquila. }\end{array}$ \\
\hline $\begin{array}{l}\text { Keijzer, } \\
\text { Tonne, } \\
\text { Sabiad, } \\
\text { Basagan, } \\
\text { Valentin, } \\
\text { Manouxd, } \\
\text { Anto, } \\
\text { Alonso, } \\
\text { huijsen, } \\
\text { Sunyer, } \\
\text { Dadvand; } \\
2019 \text { (35) }\end{array}$ & $\begin{array}{l}\text { Investigar la } \\
\text { asociación entre } \\
\text { el entorno natural } \\
\text { y la disminución } \\
\text { del } \\
\text { funcionamiento } \\
\text { físico en adultos } \\
\text { mayores. }\end{array}$ & $\begin{array}{l}5759 \\
\text { participantes de } \\
50 \text { a } 74 \text { años de } \\
\text { edad al inicio del } \\
\text { estudio en Reino } \\
\text { Unido. }\end{array}$ & $\begin{array}{l}\text { Estudio longitudinal de } \\
2002-2013 \text {, se basó en } \\
\text { el estudio Whitehall II, } \\
\text { una cohorte en curso } \\
\text { establecida en } 1985 .\end{array}$ & $\begin{array}{l}\text { Mayor verdor } \\
\text { residencial circundante } \\
\text { y proximidad a } \\
\text { entornos naturales } \\
\text { (espacios verdes y } \\
\text { azules combinados) se } \\
\text { asociaron con } \\
\text { disminución más lenta } \\
\text { (10 años) en la } \\
\text { velocidad de caminata } \\
\text { y fuerza de agarre. }\end{array}$ \\
\hline $\begin{array}{l}\text { Portegijs, T } \\
\text { sai , Ranta } \\
\text { nen, Rant } \\
\text { akokko; } \\
2016(36)\end{array}$ & $\begin{array}{l}\text { Determinar si salir } \\
\text { al exterior y } \\
\text { moverse a través } \\
\text { de áreas verdes } \\
\text { se asocia con } \\
\text { mayor actividad } \\
\text { física en personas } \\
\text { mayores }\end{array}$ & $\begin{array}{l}174 \text { individuos } \\
\text { de } 75 \text { a } 90 \text { años } \\
\text { de edad, que } \\
\text { viven en los } \\
\text { municipios de } \\
\text { Muurame y } \\
\text { Jyväskylä en } \\
\text { Finlandia } \\
\text { central. }\end{array}$ & $\begin{array}{l}\text { Estudio longitudinal; se } \\
\text { utilizó un acelerómetro } \\
\text { durante } 7 \text { días para } \\
\text { medir la actividad física } \\
\text { y completaron un diario } \\
\text { que incluye el área } \\
\text { verde más grande } \\
\text { alcanzada. }\end{array}$ & $\begin{array}{l}\text { Las personas mayores } \\
\text { son más activas } \\
\text { físicamente en los días } \\
\text { que salen al aire libre, } \\
\text { moverse por el } \\
\text { vecindario y la ciudad } \\
\text { aumenta aún más su } \\
\text { nivel de actividad } \\
\text { física. }\end{array}$ \\
\hline $\begin{array}{l}\text { Stride, } \\
\text { Cranney, } \\
\text { Scott, Hua; } \\
2017 \text { (37) }\end{array}$ & $\begin{array}{l}\text { Evaluar el uso, } \\
\text { intención de uso, } \\
\text { motivadores, } \\
\text { frecue n c i a, } \\
\text { preferencia de } \\
\text { uso, barreras y } \\
\text { facilitadores de un } \\
\text { gimnasio al aire } \\
\text { libre en adultos } \\
\text { mayores. }\end{array}$ & $\begin{array}{l}438 \\
\text { participantes de } \\
50 \text { a } 91 \text { años de } \\
\text { edad, los datos } \\
\text { se extrajeron de } \\
\text { un estudio que } \\
\text { investiga el uso } \\
\text { del parque y la } \\
\text { actividad física } \\
\text { en la reserva } \\
\text { Arthur Byrne, } \\
\text { Maroubra, } \\
\text { Sydney, } \\
\text { Australia. }\end{array}$ & $\begin{array}{l}\text { Estudio longitudinal, la } \\
\text { recolección de datos } \\
\text { fueron en dos } \\
\text { momentos, } \\
\text { inmediatamente } \\
\text { después de la } \\
\text { instalación del gimnasio } \\
\text { al aire libre (221), y de } \\
\text { tres a } 9 \text { meses de } \\
\text { seguimiento (217). }\end{array}$ & $\begin{array}{l}\text { Los gimnasios al aire } \\
\text { libre son una forma } \\
\text { aceptable de actividad } \\
\text { física para los adultos } \\
\text { mayores, los } \\
\text { facilitadores son la } \\
\text { sombra, diferentes } \\
\text { tipos de equipos y } \\
\text { clases que fomenten la } \\
\text { interacción social. Las } \\
\text { barreras para realizar } \\
\text { actividad física en el } \\
\text { gimnasio al aire libre } \\
\text { fueron proximidad, } \\
\text { desagrado de los } \\
\text { gimnasios, uso de los } \\
\text { equipos por niños y } \\
\text { tiempo. }\end{array}$ \\
\hline
\end{tabular}


REYES-RINCÓN, CAMPOS-USCANGA BENEFICIOS DE LA ACTIVIDAD FÍSICA EN ESPACIOS NATURALES SOBRE LA SALUD DEL ADULTO MAYOR

\begin{tabular}{|c|c|c|c|c|}
\hline $\begin{array}{l}\text { Zijlema, } \\
\text { Stasinskad } \\
\text {, Blake, } \\
\text { Dirgawai, } \\
\text { Flickerf, } \\
\text { Yeapg, } \\
\text { Golledgeh, } \\
\text { Hankey, } \\
\text { ieuwenhuij } \\
\text { sen, } \\
\text { Heyworth; } \\
2019 \text { (38) }\end{array}$ & $\begin{array}{l}\text { Estudiar la } \\
\text { asociación entre } \\
\text { los ambientes } \\
\text { naturales al aire } \\
\text { libre y la } \\
\text { mortalidad por } \\
\text { todas las causas } \\
\text { y causas } \\
\text { específicas en } \\
\text { hombres } \\
\text { mayores. }\end{array}$ & $\begin{array}{l}9218 \text { hombres } \\
\text { de } 65 \text { años o } \\
\text { más que viven } \\
\text { en Perth, } \\
\text { Australia } \\
\text { Occidental. }\end{array}$ & $\begin{array}{l}\text { Estudio longitudinal duró } \\
\text { de 1996-2014; se } \\
\text { utilizaron datos del } \\
\text { Health In Men Study } \\
\text { (HIMS), una cohorte } \\
\text { comunitaria de hombres } \\
\text { mayores. }\end{array}$ & $\begin{array}{l}\text { Los hombres que } \\
\text { habitaban espacios } \\
\text { con mayor nivel de } \\
\text { verdor residencial } \\
\text { circundante mostraron } \\
\text { menor riesgo de } \\
\text { mortalidad en } \\
\text { comparación con } \\
\text { aquellos con menos } \\
\text { espacios verdes. }\end{array}$ \\
\hline $\begin{array}{l}\text { Zhu, Bai, } \\
\text { Wu, Yan, } \\
\text { Tang, } \\
\text { Zeng, } \\
\text { James; } \\
2019 \text { (39) }\end{array}$ & $\begin{array}{l}\text { Explorar la } \\
\text { asociación entre } \\
\text { el verdor y la } \\
\text { mortalidad en } \\
\text { adultos mayores. }\end{array}$ & $\begin{array}{l}23754 \text { individuos } \\
\text { mayores de } 65 \\
\text { años de edad } \\
\text { pertenecientes a } \\
22 \text { de } 31 \\
\text { provincias de } \\
\text { China. }\end{array}$ & $\begin{array}{l}\text { Estudio de cohorte } \\
\text { prospectivo, de una } \\
\text { duración de 2000-2014; } \\
\text { se utilizó una encuesta } \\
\text { qué se aplicó cada dos } \\
\text { años para obtener los } \\
\text { determinantes de salud. } \\
\text { La exposición al verdor } \\
\text { se calculó a través del } \\
\text { índice de vegetación, la } \\
\text { evaluación de la } \\
\text { mortalidad por todas las } \\
\text { causas se obtuvo por } \\
\text { información de los } \\
\text { familiares. }\end{array}$ & $\begin{array}{l}\text { Una mayor exposición } \\
\text { al verdor se asoció con } \\
\text { tasas más bajas de } \\
\text { mortalidad no } \\
\text { accidental. }\end{array}$ \\
\hline $\begin{array}{l}\text { Wood, } \\
\text { Smyth; } \\
2019(40)\end{array}$ & $\begin{array}{l}\text { Examinar los } \\
\text { beneficios de la } \\
\text { exposición de la } \\
\text { naturaleza y el } \\
\text { ejercicio verde } \\
\text { durante la niñez } \\
\text { sobre la edad } \\
\text { adulta. }\end{array}$ & $\begin{array}{l}45 \text { individuos } \\
\text { sanos } \geq 50 \text { años } \\
\text { de edad. }\end{array}$ & $\begin{array}{l}\text { Estudio piloto, se utilizó } \\
\text { un cuestionario para } \\
\text { evaluar el nivel de } \\
\text { estrés; monitor } \\
\text { guardaespaldas } \\
\text { Firstbeat para medir la } \\
\text { frecuencia cardiaca } \\
\text { (FC), se usó un diario } \\
\text { dónde los participantes } \\
\text { registraron sus } \\
\text { actividades del día, hora } \\
\text { de dormir y de } \\
\text { despertar. }\end{array}$ & $\begin{array}{l}\text { La exposición a la } \\
\text { naturaleza y ejercicio } \\
\text { verde durante la niñez } \\
\text { se asoció } \\
\text { positivamente con una } \\
\text { mayor exposición, } \\
\text { participación, } \\
\text { conectividad, y } \\
\text { menores porcentajes } \\
\text { de estrés en la edad } \\
\text { adulta. }\end{array}$ \\
\hline
\end{tabular}

\section{Hallazgos relevantes}

En la mayoría de investigaciones con adultos mayores la opción de realizar actividad física en espacios naturales es la más elegida por este grupo poblacional generándoles bienestar psicosocial, además el tener contacto con espacios naturales aumenta la probabilidad de ser personas activas y lograr una mejor calidad de vida.

Son pocos los estudios que han tratado de explicar el efecto que tiene la actividad física en espacios naturales sobre la salud física en este grupo, sin embargo, en términos generales los resultados sugieren que el ejercicio verde es una buena forma para ser social y 
físicamente activos, manteniendo la autosuficiencia a través de una mejor calidad de vida en la vejez.

\section{DiscuSIÓN Y CONCLUSIONES}

Aunque desde algunas perspectivas se considera la etapa de vejez como sinónimo de dependencia, inactividad, aislamiento, enfermedad y decrepitud, esto tiene gran relación con la falta de buenos hábitos que traen como consecuencia una serie de dificultades que impiden disfrutar de una vejez plena y obstaculizan el desarrollo óptimo de sus actividades, posicionando al adulto mayor en un marco social heterónomo exigente de cuidados de familiares o agentes externos. Por otra parte, este sector de la población vive aislamiento social y exclusión, en un contexto de escasa protección institucional y profundas desigualdades sociales que se observan tanto en entornos rurales como urbanos (41). Queda claro que la vejez es una parte natural de la vida, es un proceso lento pero dinámico que depende de muchas influencias internas y externas generado en la mayoría de los casos por los estilos de vida (42).

Aunque estudios previos sugieren que ejercitarse al aire libre provoca mayor bienestar psicosocial, muy pocos estudios comprueban el beneficio de ejercitarse en espacios naturales en términos de promoción de la salud física y tratamiento de diferentes enfermedades.

Los hallazgos del presente trabajo demuestran que el ejercicio verde puede ser una ruta importante para la instauración de estilos de vida más saludables que repercutan en otras esferas de la vida del adulto mayor con especial énfasis en la salud y el bienestar. Lo que se sustenta con la evidencia presentada que indica que el contacto con espacios naturales en la vejez genera un aumento en la posibilidad de realizar actividad física y disminuir la tasa de declive cognitivo (32), así como fortalecer diferentes aspectos como seguridad en sí mismos, cohesión social, conexión con la naturaleza, bienestar psicosocial (30), mejora en la función física y psicológica (27) y disminución en la tasa de mortalidad (37).

Las premisas expuestas con antelación representan el punto de encuentro de diversos estudios resaltando que tener mayor contacto con espacios naturales genera beneficios a la salud mental así como aumento en la vitalidad (43), mejor bienestar psicológico (44) y mayor disfrute e intención futura de participación (45). Esto permite que el ejercicio verde se constituya en una estrategia para cumplir con los criterios que establece la OMS y que los estilos de vida saludables en el adulto mayor sean una realidad. 
No obstante, los adultos mayores identifican algunas barreras para realizar ejercicio verde entre las que destacan la inseguridad que vive la sociedad actual, por lo que es de suma importancia que se implementen estrategias para mejorar vigilancia en las calles y alumbrado público, así como de combate a la delincuencia que posibiliten ambientes seguros para la práctica de estas actividades. Los gobiernos requieren hacer una inversión en la conservación de espacios naturales combinando las obras producto de la ingeniería y el disfrute de lo que regala la naturaleza (20).

Con el continuo crecimiento de las poblaciones de la tercera edad en las sociedades modernas, deviene la necesidad de buscar formas factibles de conservar y mejorar las habilidades funcionales de las personas al envejecer, ayudarles a ser personas independientes en la comunidad y, fundamentalmente, mejorar su calidad de vida.

Dado que la presente investigación tiene algunas limitantes como falta de control en el rango de edad de los participantes, escasa información sobre el tiempo de exposición a estos espacios naturales, poca información sobre el efecto positivo de ejercitarse en espacios azules y la mayoría de los estudios son de tipo transversal, sería conveniente realizar un estudio de tipo longitudinal dónde se comprueben integradamente los beneficios físicos, mentales y sociales de la actividad física en espacios naturales sobre este sector poblacional. También convendría contar con instrumentos validados y adaptados a distintos contextos que permitan hacer estudios a nivel macro y llegar a generalizaciones para la implementación de estrategias. 


\section{BiBLIOGRAFÍA}

1. Organización de las Naciones Unidas. (2019). Envejecimiento. Consulta 20 febrero, 2019, de la Wold Wide Web: https://www.un.org/es/sections/ issues-depth/ageing/index.html

2. Organización Mundial de la Salud. (2018). Envejecimiento y salud. Consulta 25 febrero, 2019, de la Wold Wide Web: https://www.who.int/es/newsroom/fact-sheets/detail/envejecimiento-y-salud

3. Organización de las Naciones Unidas. (2017). World population prospects. Consulta 28 Febrero, 2019, de la Wold Wide Web: https://esa.un.org/ unpd/wpp/Publications/Files/WPP2017_KeyFindings.pdf

4. Organización Mundial de la Salud. (2019). El envejecimiento. Consulta 2 marzo, 2019, de la Wold Wide Web: https://www.who.int/topics/ ageing/es/

5. Chalapud-Narváez, L. M., \& Escobar-Almario, A. (2017). "Actividad física para mejorar fuerza y equilibrio en el adulto mayor". Universidad y Salud, 19, 94-101. Doi: https://doi.org/10.22267/rus. 171901.73

6. Roberts, C. E., Phillips, L. H., Cooper, C. L., Gray, S., \& Allan, J. L. (2017). Effect of Different Types of Physical Activity on Activities of Daily Living in Older Adults: Systematic Review and Meta-Analysis. [Meta-Analysis Review Systematic Review]. J Aging Phys Act, 25(4), 653-670. doi: 10.1123/japa.2016-0201

7. Castrogiovanni, P., Trovato, F. M., Szychlinska, M. A., Nsir, H., Imbesi, R., \& Musumeci, G. (2016). The importance of physical activity in osteoporosis. From the molecular pathways to the clinical evidence. [Review]. Histol Histopathol, 31(11), 1183-1194. doi: 10.14670/HH-11-793

8. Fernandes, M. R., \& Barreto, W. D. R. J. (2017). Association between physical activity and vitamin D: A narrative literature review. [Review]. Rev Assoc Med Bras (1992), 63(6), 550-556. doi: 10.1590/1806-9282.63.06.550

9.Triguero-Mas, M., Donaire-Gonzalez, D., Seto, E., Valentin, A., Martinez, D., Smith, G., . . Nieuwenhuijsen, M. J. (2017). Natural outdoor environments and mental health: Stress as a possible mechanism. [Research Support, Non-U.S. Gov't]. Environ Res, 159, 629-638. doi: 10.1016/j.envres. 2017.08.048

10. United States Environmental Protection Agency. (2017). What is Open Space/Green Space? Consulta. 3 marzo, 2019, de la Wold Wide web: https://www3.epa.gov/region1/eco/uep/ openspace.html

11. Grellier, J., White, M. P., Albin, M., Bell, S., Elliott, L. R., Gascon, M., . . Fleming, L. E. (2017). BlueHealth: a study programme protocol for mapping and quantifying the potential benefits to public health and well-being from Europe's blue spaces. [Research Support, Non-U.S. Gov't]. BMJ Open, 7(6). doi: 10.1136/bmjopen-2017-016188

12. Houlden, V., Weich, S., \& Jarvis, S. (2017). A cross-sectional analysis of green space prevalence and mental wellbeing in England. BMC Public Health, 17(1), 460. doi: 10.1186/s12889-017-4401$\mathrm{x}$

13. Shanahan, D. F., Franco, L., Lin, B. B., Gaston, K. J., \& Fuller, R. A. (2016). The Benefits of Natural Environments for Physical Activity. Sports Med, 46(7), 989-995. doi: 10.1007/s40279-016-0502-4

14. Chang, P. J., \& Bae, S. Y. (2017). Positive Emotional Effects of Leisure in Green Spaces in Alleviating Work-Family Spillover in Working Mothers. Int J Environ Res Public Health, 14(7). 757. doi: 10.3390/ijerph14070757

15. Vanaken, G. J., \& Danckaerts, M. (2018). Impact of Green Space Exposure on Children's and Adolescents' Mental Health: A Systematic Review. [Systematic Review]. Int J Environ Res Public Health, 15(12). 2668. doi: 10.3390/ijerph15122668

16. Britton, E., Kindermann, G., Domegan, C., \& Carlin, C. (2018). Blue care: a systematic review of blue space interventions for health and wellbeing. Health Promot Int. 103. doi: 10.1093/heapro/day103

17. Lawton, E., Brymer, E., Clough, P., \& Denovan, A. (2017). The Relationship between the Physical Activity Environment, Nature Relatedness, Anxiety, and the Psychological Well-being Benefits of Regular Exercisers. Front Psychol, 8, 1058. doi: 10.3389/fpsyg.2017.01058

18. Rogerson, M., Gladwell, V. F., Gallagher, D. J., \& Barton, J. L. (2016). Influences of Green Outdoors versus Indoors Environmental Settings on Psychological and Social Outcomes of Controlled Exercise. [Research Support, Non-U.S. Gov't]. Int J Environ Res Public Health, 13(4), 363. doi: 10.3390/ijerph13040363

19. Thompson Coon, J., Boddy, K., Stein, K., Whear, R., Barton, J., \& Depledge, M. H. (2011). Does participating in physical activity in outdoor natural environments have a greater effect on physical and mental wellbeing than physical activity indoors? A systematic review. [Research Support, Non-U.S. Gov't Review Systematic Review]. Environ Sci Technol, 45(5), 1761-1772. doi: 10.1021/ es102947t

20. Selby, S., Hayes, C., O'Sullivan, N., O'Neil, A., \& Harmon, D. (2018). Facilitators and barriers to green exercise in chronic pain. Ir J Med Sci. 1-6 doi: 10.1007/s11845-018-1923-x

21. Calogiuri, G., \& Elliott, L. R. (2017). Why Do People Exercise in Natural Environments? Norwegian Adults' Motives for Nature-, Gym-, and SportsBased Exercise. Int J Environ Res Public Health, 14(4). 377. doi: 10.3390/ijerph14040377 
22. Calogiuri, G., Patil, G. G., \& Aamodt, G. (2016). Is Green Exercise for All? A Descriptive Study of Green Exercise Habits and Promoting Factors in Adult Norwegians. Int J Environ Res Public Health, 13(11), 1165. doi: 10.3390/ijerph13111165

23. Pleson, E., Nieuwendyk, L. M., Lee, K. K., Chaddah, A., Nykiforuk, C. I., \& Schopflocher, D. (2014). Understanding older adults' usage of community green spaces in Taipei, Taiwan. [Research Support, Non-U.S. Gov't]. Int J Environ Res Public Health, 11(2), 1444-1464. doi: 10.3390/ ijerph110201444

24. Finlay, J., Franke, T., McKay, H., \& Sims-Gould, J. (2015). Therapeutic landscapes and wellbeing in later life: Impacts of blue and green spaces for older adults. [Research Support, Non-U.S. Gov't]. Health Place, 34, 97-106. doi: 10.1016/j.healthplace.2015.05.001

25. Portegijs, E., Keskinen, K. E., Tsai, L. T., Rantanen, T., \& Rantakokko, M. (2017). Physical Limitations, Walkability, Perceived Environmental Facilitators and Physical Activity of Older Adults in Finland. Int J Environ Res Public Health, 14(3), 333. doi: 10.3390/ijerph14030333

26. Astell-Burt, T., Feng, X., \& Kolt, G. S. (2014). Green space is associated with walking and moderate-to-vigorous physical activity (MVPA) in middleto-older-aged adults: findings from 203883 Austra- $^{-}$ lians in the 45 and Up Study. Br J Sports Med, 48(5), 404-406. doi: 10.1136/bjsports-2012-092006

27. Harada, K., Lee, S., Bae, S., Suzuki, T., \& Shimada, H. (2017). Objectively-measured outdoor time and physical and psychological function among older adults. [Multicenter Study Randomized Controlled Trial]. Geriatr Gerontol Int, 17(10), 1455-1462. doi: 10.1111/ggi.12895

28. Wu, Y. T., Jones, N. R., van Sluijs, E. M., Griffin, S. J., Wareham, N. J., \& Jones, A. P. (2016). Perceived and Objectively Measured Environmental Correlates of Domain-Specific Physical Activity in Older English Adults. [Research Support, NonU.S. Gov't]. J Aging Phys Act, 24(4), 599-616. doi: 10.1123/japa.2015-0241

29.- Cassarino M, Bantry-White E, Setti A. (2019). Cognitive and Sensory Dimensions of Older People's Preferences of Outdoor Spaces for Walking: A Survey Study in Ireland. International journal of environmental research and public health, 16(8), 1660-4601. doi:10.3390/ijerph16081340

30. Silva Piñeiro, Roberto, \& Mayán Santos, José Manuel. (2016). Beneficios psicológicos de un programa proactivo de ejercicio físico para personas mayores. Escritos de Psicología (Internet), 9(1), 24-32. doi: 10.5231/psy.writ. 2015.2212

31. Lacharite-Lemieux, M., Brunelle, J. P., \& Dionne, I. J. (2015). Adherence to exercise and affective responses: comparison between outdoor and indoor training. [Comparative Study Randomized Controlled Trial Research Support, Non-U.S. Gov't]. Menopause, 22(7), 731-740. doi: 10.1097/ GME.0000000000000366

32. King, A. C., Salvo, D., Banda, J. A., Ahn, D. K., Chapman, J. E., Gill, T. M., . . Frank, L. D. (2017). Preserving older adults' routine outdoor activities in contrasting neighborhood environments through a physical activity intervention. Prev Med, 96, 87-93. doi: 10.1016/j.ypmed.2016.12.049

33. Cherrie, M. P. C., Shortt, N. K., Mitchell, R. J., Taylor, A. M., Redmond, P., Thompson, C. W., . . . Pearce, J. R. (2018). Green space and cognitive ageing: A retrospective life course analysis in the Lothian Birth Cohort 1936. [Research Support, Non-U.S. Gov't]. Soc Sci Med, 196, 56-65. doi: 10.1016/j.socscimed.2017.10.038

34. Neale, C., Aspinall, P., Roe, J., Tilley, S., Mavros, P., Cinderby, S., .. Thompson, C. W. (2017). Correction to: The Aging Urban Brain: Analyzing Outdoor Physical Activity Using the Emotiv Affectiv Suite in Older People. [Published Erratum]. J Urban Health, 94(6), 881. doi: 10.1007/ s11524-017-0209-3

35.de Keijzer, C., Tonne, C., Sabia, S., Basagana, X., Valentin, A., Singh-Manoux, A., . . . Dadvand, P. (2019). Green and blue spaces and physical functioning in older adults: Longitudinal analyses of the Whitehall II study. Environ Int, 122, 346-356. doi: 10.1016/j.envint.2018.11.046

36. Tsai, L. T., Rantakokko, M., Viljanen, A., Saajanaho, M., Eronen, J., Rantanen, T., \& Portegijs, E. (2016). Associations Between Reasons to Go Outdoors and Objectively-Measured Walking Activity in Various Life-Space Areas Among Older People. [Research Support, Non-U.S. Gov't]. J Aging Phys Act, 24(1), 85-91. doi: 10.1123/japa.2014-0292

37. Stride, V., Cranney, L., Scott, A., \& Hua, M. (2017). Outdoor gyms and older adults - acceptability, enablers and barriers: a survey of park users. Health Promot J Austr, 28(3), 243-246. doi: 10.1071/HE16075

38. Zijlema, W. L., Stasinska, A., Blake, D., Dirgawati, M., Flicker, L., Yeap, B. B., . . . Heyworth, J. (2019). The longitudinal association between natural outdoor environments and mortality in 9218 older men from Perth, Western Australia. Environ Int, 125, 430-436. doi: 10.1016/j.envint. 2019.01.075

39. Ji, J. S., Zhu, A., Bai, C., Wu, C. D., Yan, L., Tang, S., . . James, P. (2019). Residential greenness and mortality in oldest-old women and men in China: a longitudinal cohort study. Lancet Planet Health, 3(1), 17-25. doi: 10.1016/ S2542-5196(18)30264-X

40. Wood CJ, Smyth N. (2019). The health impact of nature exposure and green exercise across the life course: a pilot study. International journal of 
environmental health research, 108(1), 95-103. doi. 10.1080/09603123.2019.1593327

41. Giraldo-Ocampo, CP, \& Cardona-Arango, D. (2010). Ser viejo en colombia tiene su costo laboral. Investigaciones Andina, 12(21), 50-59. Recuperado de: http://www.scielo.org.co/scielo.php? script=sci_arttext\&pid=S0124-8146201000020000 $5 \& \operatorname{lng}=$ en\&tlng=es.

42. Organización Mundial de la Salud. (2019). El envejecimiento saludable. Consulta 2 marzo, 2019, de la Wold Wide Web: https://fiapam.org/wp-content/uploads/2012/10/oms-envejecimiento-02.pdf

43. van den Berg, M., van Poppel, M., van Kamp, I., Andrusaityte, S., Balseviciene, B., Cirach, M., . . . Maas, J. (2016). Visiting green space is associated with mental health and vitality: A cross- sectional study in four european cities. [Research Support, Non-U.S. Gov't]. Health Place, 38, 8-15. doi: 10.1016/j.healthplace.2016.01.003

44. Astell-Burt, T., Feng, X., \& Kolt, G. S. (2013). Mental health benefits of neighbourhood green space are stronger among physically active adults in middle-to-older age: evidence from 260,061 Australians. Prev Med, 57(5), 601-606. doi: 10.1016/j.ypmed.2013.08.017

45. Krinski, K., Machado, D. G. S., Lirani, L. S., DaSilva, S. G., Costa, E. C., Hardcastle, S. J., \& Elsangedy, H. M. (2017). Let's Walk Outdoors! Self-Paced Walking Outdoors Improves Future Intention to Exercise in Women With Obesity. J Sport Exerc Psychol, 39(2), Ed. Board. 145-157. doi: 10.1123/jsep.2016-0220 